\title{
Inseminação artificial e acompanhamento da gestação em uma cadela da raça Bull Terrier com histórico de agressividade: relato de caso
}

Kianne Silva Monteiro ${ }^{1}$, Luana Janetizk Bergmann², Bruna Conceição Ferreira ${ }^{3}$, Gabriel Marchesi Lira ${ }^{4}$, Thamires Fernanda Ramalho Marques ${ }^{5}$, Taize Cristina Fonseca ${ }^{6}$, Elizabeth Beraldo ${ }^{7}$, Luis David Solis Murgas ${ }^{8 *}$

DOI: https://doi.org/10.35699/2447-6218.2020.16292

\begin{abstract}
Resumo
O objetivo desse trabalho é relatar uma inseminação artificial (IA) e posterior acompanhamento gestacional realizados em uma cadela da raça Bull Terrier com histórico de agressividade durante o estro. Foi atendida uma cadela da raça Bull Terrier com 14 meses de idade com sinais aparentes de estro, que demonstrou agressividade e intolerância à aproximação do macho na monta natural, sendo sugerido realizar a IA. A citologia vaginal confirmou a fase de estro, com mais de $80 \%$ de células queratinizadas anucleadas. O sêmen do macho doador foi coletado por manipulação digital e posteriormente analisado, apresentando qualidade em todos os parâmetros espermáticos avaliados. Foi realizada inseminação artificial intravaginal com sêmen fresco, e o mesmo procedimento foi repetido 48 horas, depois de uma segunda citologia vaginal confirmando que o animal ainda estava em estro. Essa repetição da IA teve o intuito de abranger melhor a duração do estro, aumentando a chance de prenhês. Após 51 dias da última IA foi realizada uma ultrassonografia para confirmar a gestação, sendo possível observar a presença de fetos vivos, cuja viabilidade foi comprovada pela presença de batimentos cardíacos entre 230 a $257 \mathrm{bpm}$. O parto ocorreu 58 dias após a última inseminação, com duração de aproximadamente oito horas e nascimento de cinco filhotes, todos vivos e saudáveis. Foi possível concluir que IA após confirmação de estro por citologia vaginal, e o acompanhamento gestacional, são ferramentas importantes da biotecnologia da reprodução, capazes de proporcionar a utilização de animais na atividade reprodutiva, em casos onde a monta natural não é possível.
\end{abstract}

Palavras-chave: Reprodução canina. Citologia vaginal. Ciclo estral.

\section{Artificial insemination and gestation follow-up in Bull Terrier dog breed with aggressiveness's history: case report}

Abstract

\footnotetext{
${ }^{1}$ Universidade Federal de Lavras. Lavras, Minas Gerais. Brasil https://orcid.org/0000-0002-4298-359X

${ }^{2}$ Universidade Federal de Lavras. Lavras, Minas Gerais. Brasil https://orcid.org/0000-0002-1067-2914

${ }^{3}$ Universidade Federal de Lavras. Lavras, Minas Gerais. Brasil https://orcid.org/0000-0002-5244-6050

${ }^{4}$ Universidade Federal de Lavras. Lavras, Minas Gerais. Brasil https://orcid.org/0000-0002-6545-2898

${ }^{5}$ Universidade Federal de Lavras. Lavras, Minas Gerais. Brasil https://orcid.org/0000-0001-6231-6631

${ }^{6}$ Universidade Federal de Lavras. Lavras, Minas Gerais. Brasil https://orcid.org/0000-0002-3912-4065

${ }^{7}$ Universidade Federal de Lavras. Lavras, Minas Gerais. Brasil https://orcid.org/0000-0002-7837-6302

${ }^{8}$ Universidade Federal de Lavras. Lavras, Minas Gerais. Brasil https://orcid.org/0000-0002-0066-7505

*Autor para correspondência : 1smurgas@ufla.br
}

Recebido para publicação em 20 de janeiro de 2020. Aceito para publicação em 28 de junho de 2020.

e-ISSN: 2447-6218 / ISSN: 2447-6218 / (c) 2009, Universidade Federal de Minas Gerais, Todos os direitos reservados. 
Monteiro, K. S. et al.

The objective of this work was to report an artificial insemination (AI) and subsequent gestational follow - up performed on a Bull Terrier bitch with a history of aggressiveness. A 14-month-old Bull Terrier was received with apparent signs of estrus, which showed aggression and intolerance to the approach of the male in the natural mating, and AI was suggested. Vaginal cytology confirmed the estrus phase, with more than $80 \%$ of anucleated keratinized cells. The semen of the male donor was collected by digital manipulation and later analyzed, presenting quality in all sperm parameters evaluated. Intravaginal artificial insemination was performed with fresh semen, and the same procedure was repeated 48 hours after a second vaginal cytology confirming that the animal was still in estrus. This repetition of AI was designed to better cover the duration of estrus, thus increasing the chance of breeding. After 51 days of the last AI, an ultrasonography was performed to confirm gestation. It was possible to observe the presence of live fetuses, whose viability was confirmed by the presence of heart beats between 230 and $257 \mathrm{bpm}$. The birthing occurred 58 days after the last insemination, lasting approximately eight hours and birth of five pups, all alive and healthy. It was possible to conclude that AI after confirmation of estrus by vaginal cytology and gestational monitoring are important tools of reproduction biotechnology capable of providing the use of animals in reproductive activity in cases where natural reproduction is not possible.

Keywords: canine reproduction, vaginal cytology, estrus cycle

\section{Introdução}

A citologia vaginal é um exame laboratorial complementar de grande utilidade no manejo reprodutivo em cadelas, importante para realização da inseminação artificial (IA) de forma correta (Mason, 2018). Durante o estro a cadela torna-se receptiva ao macho, a vulva torna-se flácida e edemaciada e na citologia vaginal, observa-se $100 \%$ das células anucleadas e queratinizadas (Vieira et al., 2012), com alto número de células, inicialmente com núcleos picnóticos, e células escamosas anucleadas que se localizam em ninhos celulares (Dar et al., 2017).

Na espécie canina a IA pode ser utilizada como um meio alternativo quando existe a impossibilidade de realização de monta natural, devido a problemas comportamentais e sanitários (Silva et al., 2003). O sêmen utilizado na IA pode ser fresco, refrigerado ou congelado (Uchoa et al., 2012 e Mason, 2018), sendo coletado, na maioria das vezes, por manipulação digital do corpo peniano do cão (Setyawan et al., 2016 e Karger et al., 2016), e avaliado quanto à alguns parâmetros, como: volume, concentração espermática, motilidade e morfologia (England et al., 2010; CBRA, 2013; Tesi et al., 2018).

A IA tem indicações veterinárias para diversas ocasiões, inclusive para casos de agressividade dos animais (Silva et al., 2003). O cão Bull Terrier, na maioria das vezes, tem um contexto de agressividade elevada manifestada quando na presença de cães que não são do seu convívio (Duffy et al., 2008). Não tem sido relatado, na literatura, alterações comportamentais, nessa raça, durante os períodos de reprodução.

Após a realização da IA, é necessário um acompanhamento gestacional, podendo ser realizado por vários métodos, sendo comumente utilizada a ultrassonografia, radiografia e palpação abdominal (Freitas \& Silva, 2008; Veiga et al., 2013). A ultrassonografia é um método não invasivo, confiável e seguro, que permite o diagnóstico da gestação, a idade gestacional, as condições dos ovários e do útero, a contagem aproximada do número de embriões ou fetos, a observação direta da atividade do coração e dos movimentos fetais, entre outros (Castro et al., 2011; Lamm \& Makloski, 2012).

O objetivo desse trabalho é relatar a IA e posterior acompanhamento gestacional em cadela com histórico de agressividade durante o estro, que inviabilizou a monta natural.

\section{Relato de caso}

Foi atendida no Laboratório de Fisiologia do Departamento de Medicina Veterinária da Universidade Federal de Lavras (UFLA), uma cadela da raça Bull Terrier, 14 meses de idade, com sinais aparentes de estro. Inicialmente foi feita uma tentativa de realizar monta natural com um macho da mesma raça, mas devido à agressividade e rejeição por parte da fêmea, a monta natural não foi concretizada.

A cadela se encontrava no segundo cio, sendo que estes eram regulares, ocorrendo a cada seis meses. Aproximadamente nove dias antes da realização da citologia vaginal foram constatados os sinais indicativos de proestro, como secreção serossanguinolenta e edema dos lábios vulvares. Foi realizada citologia vaginal com auxílio de uma zaragatoa preparada com solução salina, introduzido dorso-cranialmente na face dorsal da comissura labial a um ângulo de $45^{\circ}$ e depois de $180^{\circ}$ para que se obtivesse material da região mais cranial da vagina (Costa, 2009). Foram realizadas de 2 a 3 revoluções com a zaragatoa, e o material coletado foi fixado em lâmina devidamente identificada, que foi posteriormente corada com corante do tipo Panótico Rápido. Após a coloração e secagem, cada lâmina foi submetida à microscopia óptica com aumento de 40x (Figura 1), sendo avaliada a porcentagem de células parabasais, intermediárias e superficiais queratinizadas (Costa, 2009). 
Figura 1 - citologia vaginal avaliada em microscopia eletrônica: fase de estro

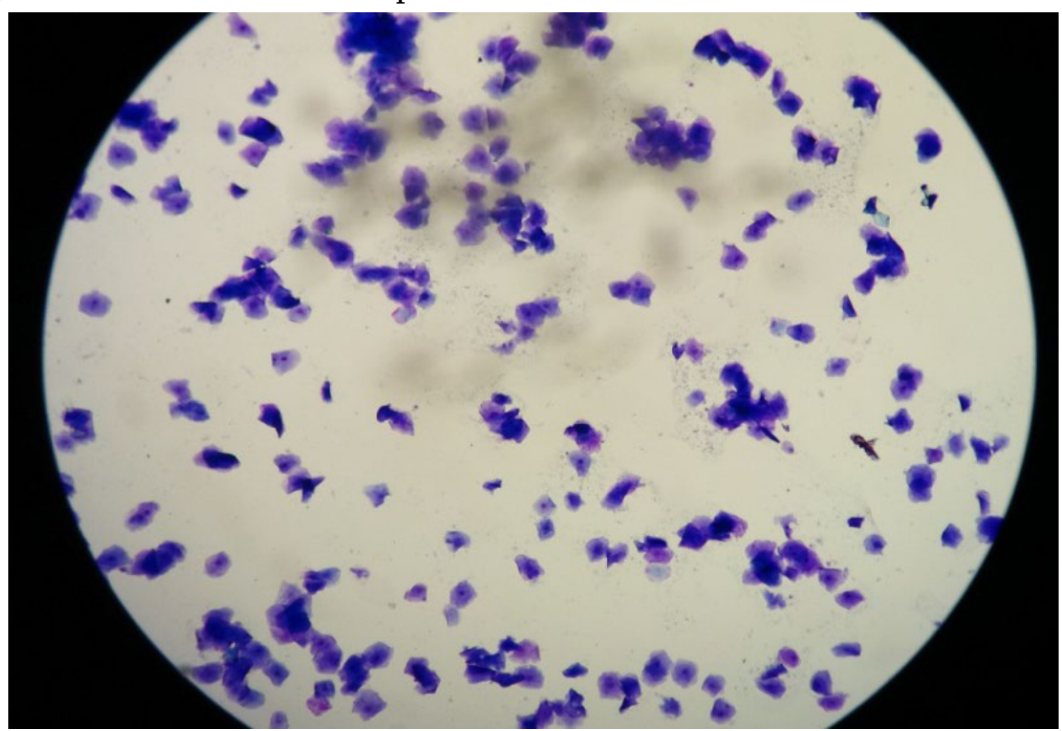

O sêmen foi coletado pelo método de manipulação digital (Setyawan et al., 2016; Karger et al., 2016) e como reprodutor foi selecionado um cão adulto e sadio

Figura 2 - coleta do sêmen para inseminação artificial da raça Bull Terrier, que já teve atividade reprodutiva anteriormente por duas vezes, por monta natural (Figura 2).

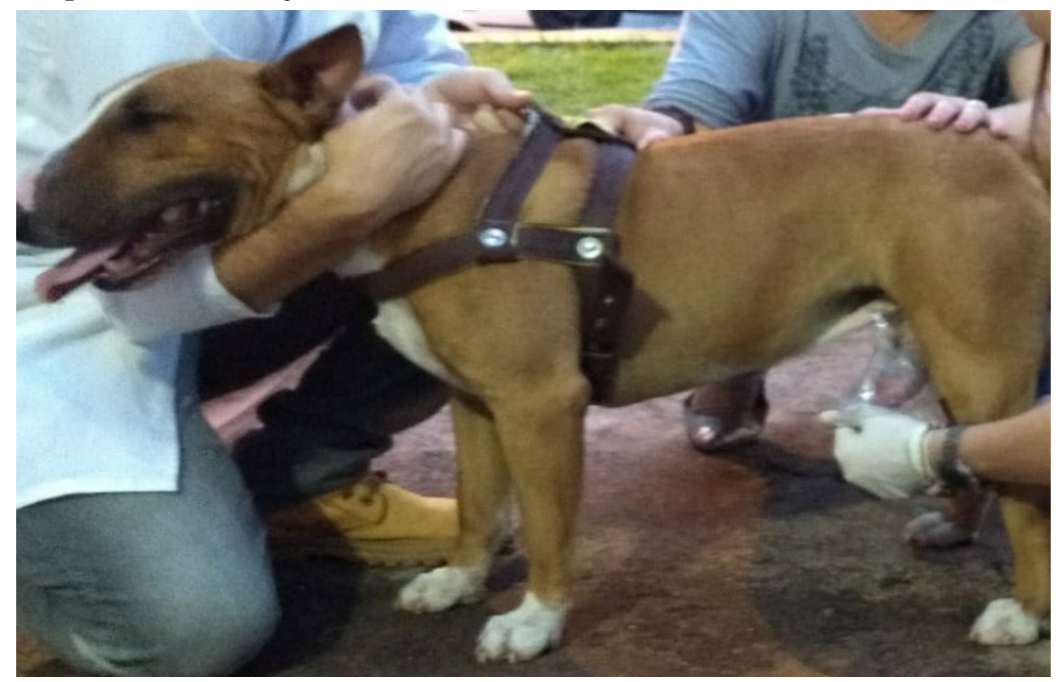

Posteriormente, foi realizada limpeza da vulva com algodão e solução de cloreto de sódio a $0,9 \%$, e utilizando como guia o dedo indicador posicionado de forma perpendicular a comissura dorsal vulvar, introduziu-se a pipeta de inseminação universal da marca IMV Technologies ${ }^{\circledR}$ na vagina da cadela na extensão de aproximadamente $15 \mathrm{~cm}$. Foi utilizada a técnica de inseminação artificial intravaginal, que consiste na deposição do sêmen à fresco, na porção cranial da vagina da cadela, por ser de fácil execução e por oferecer bons resultados de um modo geral (Mason, 2018).

A seringa contendo o sêmen foi acoplada à extremidade posterior da pipeta e aplicado lentamente. Após realização da IA, os membros pélvicos foram mantidos elevados durante aproximadamente 15 minutos, visando prevenir o refluxo de sêmen. Entretanto, durante esse procedimento a fêmea demonstrou episódios de agres- sividade contra o inseminador e, após ser acalmada pela presença do tutor, foi possível prosseguir, em um segundo momento, com a inseminação artificial.

O mesmo procedimento foi repetido após 48 horas, como forma de garantir a entrada de conteúdo espermático suficiente no interior do útero durante o período de estro, aumentando assim a chance de prenhês. No último dia de procedimento a citologia vaginal já demonstrava maior porcentagem de células intermediárias (26\%) e aumento de células queratinizadas com núcleo (34\%) e, por isso, a inseminação não foi realizada nesse momento.

Para acompanhamento gestacional, um exame de ultrassonografia foi realizado no Hospital Veterinário da Universidade Federal de Lavras, 51 dias após a última inseminação (Figura 3). O exame foi realizado em apa- 
Monteiro, K. S. et al.

relho Toshiba Aplio 300, transdutor linear (8-18 MHz). Anteriormente ao exame de ultrassonografia a fêmea apresentou-se agressiva com o próprio tutor, e houve relato do mesmo dizendo que o comportamento da fêmea se tornou mais instável durante a gestação. Através da ultrassonografia realizada, foi possível observar a presença de fetos vivos cuja viabilidade foi comprovada pela presença de batimentos cardíacos entre 230 a 257 bpm.

Figura 3 - realização do exame ultrassonográfico para acompanhamento gestacional

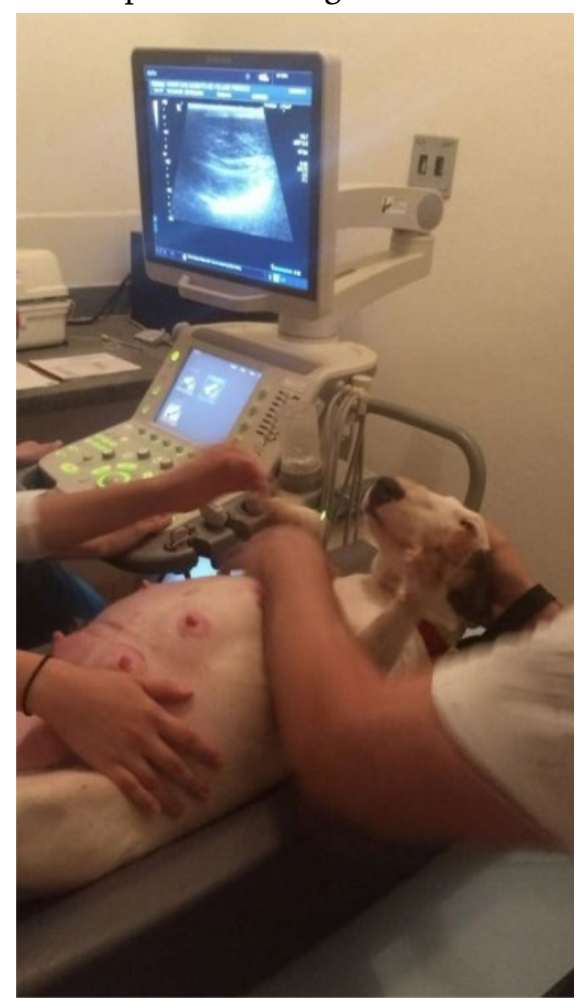

Sete dias após a realização do exame de ultrassonografia a cadela apresentou sinais característicos de início de parto, como inquietação, procura de ninho, diminuição de apetite, edema mamário, produção de leite e liberação de muco tampão pela vulva. Além disso, não permitia a aproximação do proprietário com sinais de agressividade. O parto ocorreu 58 dias após a última inseminação. A cadela começou a apresentar contrações abdominais poucas horas antes do nascimento do primeiro filhote, e a duração do parto foi de aproximadamente oito horas. Nasceram cinco filhotes, todos saudáveis e viáveis, entretanto, nas primeiras 48 horas após o parto, todos eles vieram a óbito, provocado pelo comportamento agressivo da mãe contra os filhotes.

\section{Resultados e discussão}

A citologia vaginal mostrou que o animal se encontrava na fase de estro, devido à presença de mais de $80 \%$ das células superficiais, $17 \%$ de intermediárias grandes e presença de hemácias. Em termos citológicos, considera-se que a cadela está em estro quando o esfregaço celular apresenta índice de cornificação superior a $80 \%$ (Mason, 2018), o que indicou que a cadela se encontrava apta à realização da inseminação artificial. É um exame necessário para se determinar o momento certo de realizar a inseminação artificial pela identificação da fase do ciclo estral (Feldman, 1996).

A IA em cães tornou-se uma prática comum na medicina veterinária, pois representa um instrumento fundamental para a melhora genética em espécies de interesse econômico e zootécnico (Uchoa, 2012). Além disso, o sêmen tem uma maior rentabilidade através da IA, permitindo que o material genético seja possa ser distribuído a um maior número de receptoras (Silva et al., 2001).

O sêmen foi coletado por manipulação digital e posteriormente analisado, apresentando motilidade $80 \%$, vigor 3 , volume ejaculado de $1,4 \mathrm{ml}$ e concentração espermática de $175 \times 10^{6}$ espermatozóides/mL, sendo esses parâmetros espermáticos correspondentes com a normalidade (CBRA, 2013). A distribuição e a sobrevida das células espermáticas no lúmen uterino dependem de uma série de fatores, como por exemplo a qualidade espermática, método de inseminação artificial utilizado, fase do ciclo estral em que a cadela se encontra, tipo de sêmen (fresco, refrigerado ou descongelado) e outras variações inerentes às características individuais do animal (Fukushima et al., 2010). Entretanto, as taxas de prenhês são maiores quando se utiliza um sêmen de qualidade, dentro dos parâmetros normais (Mason, 2018).

O exame ultrassonográfico é muito usado para diversos fins de diagnósticos como a confirmação de gestação, visualização de fluxo sanguíneo, verificação de algum defeito fetal congênito ou presença de anormalidades endometriais, entre outros (Davidson \& Baker, 2009; Lamm \& Makloski, 2012). De acordo com Freitas e Silva (2008) os batimentos fetais são detectados apenas durante os quinze dias finais da gestação. A média da frequência dos batimentos cardíacos de fetos da espécie canina é de $230 \mathrm{bpm}$, com cerca de 214bpm aos 24-25 dias, e de 238 bpm por volta de 40 dias. No entanto, há redução desses valores quando o momento do parto se aproxima (Lopate, 2008).

Observou-se líquido e anexo fetais dentro da normalidade ultrassonográfica, segundo organogênese fetal. Foi possível identificar diferenciação entre cavidade torácica e abdominal, estruturas ósseas formando sombreamento acústico, pelve renal discretamente dilatada, alças intestinais com presença de peristaltismo focal e estratificação parietal. Segundo as fórmulas gestacionais (idade gestacional $=($ DBP $\times 15)+20)$ a idade gestacional foi de aproximadamente 53 a 59 dias.

A IA em cães tornou-se uma prática comum na medicina veterinária, pois representa um instrumento fundamental para a melhora genética em espécies de interesse econômico e zootécnico (Uchoa, 2012). Além disso, o sêmen tem uma maior rentabilidade através da IA, 
permitindo que o material genético possa ser distribuído a um maior número de receptoras (Silva et al., 2001).

A agressividade pode ser observada em cães da raça Bull Terrier (Duffy et al., 2008), sendo uma característica intrínseca da raça, e não somente do animal atendido nesse caso. Esse animal teve momentos de agressividade no estro devido a presença do macho, no final da gestação e no pós-parto, sendo que anteriormente não havia relato do tutor de um comportamento agressivo. Entretanto, em fêmeas da raça com essa característica agressiva, deve-se tomar um cuidado especial com os filhotes após o nascimento, garantindo sua segurança. É importante também pensar em formas alternativas de controle dessa agressividade, como um manejo mais adequado dos animais, seleção de animais mais dóceis e uso de estratégias de adestramento e socialização.

\section{Conclusão}

Percebe-se que, nesse caso onde a agressividade, no momento do estro, inviabilizou a monta natural, a IA apresenta-se como uma ferramenta simples e eficiente, sendo aplicável em casos onde a monta natural é inviável, possibilitando a utilização do animal na vida reprodutiva. Entretanto, o comportamento de agressividade deve ser avaliado individualmente para o sucesso na reprodução e também para a sobrevida dos filhotes.

\section{Referências}

Castro, V. M. et al. 2011. Acompanhamento da Gestação em Cadelas pelo exame Ultrassonográfico. Revisão de Literatura. Veterinária e Zootecnia, 18(1): 9-18. Disponível em: https://repositorio.unesp.br/ handle/11449/141240.

Colégio Brasileiro de Reprodução Animal - CBRA. 2013. Manual para Exame Andrológico e Avaliação do Sêmen Animal. 3 ed. Belo Horizonte, Brasil.

Costa, E. C. F. et al. 2009. Estimativa da fase do ciclo estral por citologia vaginal em cadelas (canis familiaris, linnaeus, 1758) da região de Ituverava-sp. Nucleus Animalium, 1(2). Doi: 10.3738/1982.2278.310.

Dar, R. R. et al. 2017. Exfoliative Vaginal Cytology (EVC) as a tool for determining breeding time in the bitch a case study. Journal of Entomology and Zoology Studies, 5(6):1446-1448. Disponível em: http://www. entomoljournal.com/archives/2017/vol5issue6/PartT/5-6-140-949.pdf.

Davidson, A. P; Baker, T. W. 2009. Reproductive ultrasound of the Bitch and Queen. Topics in Companion Animal Medicine, 24(2): 55-63. Doi: https://doi.org/10.1053/j.tcam.2008.11.002.

Duffy, D. L. et al. 2008. Breed differences in canine aggression. Applied Animal Behaviour Science, 114: 441-460. Doi: 10.1016/j.applanim.2008.04.006.

England, G. C. W. et al. 2010. Heritability of semen characteristics in dogs. Theriogenology, 74(7): 1136-1140. Doi: http://dx.doi.or$\mathrm{g} / 10.1016 / \mathrm{j}$.theriogenology.2010.05.012.

Feldman, E. C.; Nelson, R. W. 1996. Breeding, Pregnancy, and Parturition. p. 547-571. In: FELDMAN, E.C.; NELSON, R.W. Canine and feline endocrinology and reproduction. Saunders Company, Philadelphia.

Freitas, J. G.; Silva, A. R. 2008. Diagnóstico de gestação em cadelas. Revista Brasileira de Reprodução Animal, 32(1): 58-66. Disponível em: http://www.cbra.org.br/pages/publicacoes/rbra/download/RB144\%20 pag58.pdf.

Fukushima, F. B.et al. 2010. Site of Intrauterine Artificial Insemination in the Bitch does not Affect Sperm Distribution within the Uterus. Reproduction in Domestic Animals, 45: 1059-1064. Doi: 10.1111/j. 1439-0531.2009.01493.x.

Karger S. et al. 2016. Prognostic value of a pre-freeze hypo-osmotic swelling test on the post-thaw quality of dog semen. Animal Reproduction Science, 166: 141-147. Doi: http://dx.doi.org/10.1016/j. anireprosci.2016.01.015.
Lamm, C. G.; Makloski, C. L. 2012. Current advances in gestation and parturition in cats and dogs. Veterinary Clinics of North America: Small Animal Practice, 42(3): 445-456. Disponível em: https://www.vetsmall. theclinics.com/article/S0195-5616(12)00011-3/abstract.

Lopate, C. 2008. Estimation of gestational age and assessment of canine fetal maturation using radiology and ultrasonography: a review. Theriogenology, 70(3): 397-402. Doi: https://doi.org/10.1016/j.theriogenology.2008.05.034.

Mason, S. J. 2018. Current review of artificial insemination in dogs. Veterinary Clinic Small Animals, 48(4): 567-580. Doi: https://doi. org/10.1016/j.cvsm.2018.02.005.

Setyawan, E. M. N., et al. 2016. Spermine reduces reactive oxygen species levels and decreases cryocapacitation in canine sperm cryopreservation. Biochemical and Biophysical Research Communication, 479(4):927-32. Doi: http://dx.doi.org/10.1016/j.bbrc.2016.08.091.

Silva, A. R. et al. 2001. Criopreservação do sêmen canino: revisão. Ciência Animal, 11(2): 119-129. Disponível em: http://www.uece.br/ cienciaanimal/dmdocuments/Artigo7.2001.2.pdf.

Silva, A. R. et al. 2003. Principais aspectos ligados à aplicação da inseminação artificial na espécie canina. Revista Portuguesa de Ciências Veterinárias, 98: 53-60. Disponível em: http://www.spcv.pt/download/ Vol98/Vol98-n546.pdf.

Tesi, M. et al. 2018. Variables affecting semen quality and its relation to fertility in the dog: A retrospective study. Theriogenology, 118:34-39. Doi: https://doi.org/10.1016/j.theriogenology.2018.05.018.

Uchoa, D. C. S. et al. 2012. Criopreservação de sêmen e inseminação artificial em cães. Ciência Animal, 22 (1). Disponível em: http://www.uece. $\mathrm{br} /$ cienciaanimal/dmdocuments/CONERA_PALESTRA\%20(10).pdf.

Veiga, C. C. P. et al. 2013. Avaliação pré-natal ultrassonográfica e radiográfica no diagnóstico de anencefalia em cão - relato de caso. Revista Brasileira de Medicina Veterinária, 35(2): 101-104. Disponível em: http://rbmv.org/index.php/BJVM/article/download/596/460.

Vieira, M. M. F. et al. 2012. Detecção do ciclo estral por meio de citologia vaginal de cadelas atendidas no hospital veterinário da Univiçosa/ Facisa. Anais do IV SIMPAC, Viçosa, MG, Brasil. Disponível em: https:// academico.univicosa.com.br/revista/index.php/RevistaSimpac/article/ download/207/369. 